\title{
Expansão decimal de números reais e conjuntos de medida total
}

\section{Decimal expansion of real numbers and sets of total measure}

\author{
Leandro Batista Morgado \\ Universidade Federal de Santa Catarina (UFSC) \\ Departamento de Matemática, Florianópolis, SC, Brasil \\ leandro.morgado@ufsc.br
}

\section{Informações do Artigo}

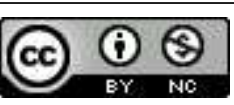

Histórico do Artigo

Submissão: 9 de novembro de 2017.

Aceite: 17 de abril de 2018.

\section{Palavras-chave}

Expansão Decimal

Teoria Ergódica

Teoria da Medida

Números Normais

Medida de Lebesgue

\section{Keywords}

Decimal Expansion

Ergodic Theory

Measure Theory

Normal Numbers

Lebesgue Measure

\begin{abstract}
Resumo
Neste artigo, abordamos questões relativas à frequência dos algarismos na expansão decimal dos números reais no intervalo $[0,1]$. Usando resultados de Teoria Ergódica e Teoria da Medida, exibiremos alguns subconjuntos de medida de Lebesgue total. Entre eles, destacamos o subconjunto dos números cujo primeiro dígito aparece novamente na expansão decimal, bem como o subconjunto dos números normais.
\end{abstract}

\begin{abstract}
In this article, we discuss some questions about the frequency of digits in decimal expansion of real numbers in the interval $[0,1]$. Using results of Ergodic Theory and Measure Theory, we present some subsets with total Lebesgue measure. Among them, we highlight the set of real numbers whose first digit appears again in decimal expansion, and the set of normal numbers.
\end{abstract}

\section{Considerações Iniciais}

A medida de Lebesgue ${ }^{1}$ de um subconjunto (mensurável) dos números reais fornece uma noção do comprimento deste subconjunto. Para um intervalo $[a, b]$, por exemplo, escrevemos $\lambda([a, b])=$ $b-a$ para denotar que a medida de Lebesgue deste intervalo vale $b-a$, e a mesma expressão vale para intervalos na forma $(a, b),[a, b)$ e $(a, b]$.

Se estamos interessados apenas em números reais pertencentes ao intervalo $[0,1]$, a medida de Lebesgue é uma medida de probabilidade, pois a medida total do espaço amostral é 1 . Nesse contexto, dizemos que a probabilidade de um número escolhido ao acaso pertencer a um subconjunto $E \subseteq[0,1]$ é dada por $\lambda(E)$. Assim, por exemplo, a probabilidade de um número escolhido ao acaso ser maior que 0,3 pode ser estimada por $\lambda((0,3,1])=0,7$, e assim sucessivamente.

\footnotetext{
${ }^{1}$ A construção e detalhes técnicos sobre a medida de Lebesgue podem ser encontrados em Castro [3], Isnard [4], Taylor [9], entre outros.
} 
Em Teoria da Medida, um fato conhecido é que, escolhido um número real ao acaso no intervalo $[0,1]$, a probabilidade de que esse número seja racional é nula. Trata-se de uma consequência direta do fato que o conjunto dos números racionais é enumerável (veja por exemplo Lima [5]). Assim, se nossa escolha for aleatória em relação à medida de Lebesgue no intervalo $[0,1]$, é quase certo que vamos escolher um número irracional. Usando linguagem técnica, dizemos que o conjunto dos números irracionais é de medida total em relação à medida de Lebesgue no intervalo $[0,1]$.

Nesta discussão, nossa intenção é verificar que essa mesma propriedade de medida total também é válida para outros subconjuntos. Vamos considerar, inicialmente, o conjunto dos números reais no intervalo $[0,1]$ com a propriedade que o primeiro dígito aparece novamente em sua expansão decimal. Posteriormente, vamos restringir ainda mais essa propriedade, exigindo que esse mesmo dígito apareça infinitas vezes na expansão decimal correspondente.

Em suma, estamos interessados em questões do tipo:

- Qual a probabilidade do dígito 7 voltar a aparecer na expansão decimal de um número contido no intervalo $\left[\frac{7}{10}, \frac{8}{10}\right)$ ?

- Qual a probabilidade do dígito 7 voltar a aparecer infinitas vezes na expansão decimal de um número contido no intervalo $\left[\frac{7}{10}, \frac{8}{10}\right)$ ?

- Qual a medida do conjunto dos números em que todos os dígitos de 0 a 9 aparecem com frequência $\frac{1}{10}$ na sua expansão decimal?

O último questionamento está relacionado com o assim denominado subconjunto dos números normais. Veremos, de forma precisa, que um número normal é caracterizado pela propriedade de que todos os possíveis blocos de $k$ algarismos aparecem com a frequência $\frac{1}{10^{k}}$ em sua expansão decimal. Em particular, todos os algarismos de 0 a 9 aparecem com a mesma frequência na expansão decimal de um número normal.

Para responder esses questionamentos, apresentando os resultados correspondentes, vamos usar ferramentas de Teoria da Medida e Teoria Ergódica, bem como uma linguagem de sistemas dinâmicos. Nesse sentido, denotando por $\{y\}$ a parte decimal de $y \in \mathbb{R}$, considere a seguinte transformação:

$$
\begin{aligned}
g:[0,1] & \rightarrow[0,1] \\
x & \mapsto\{10 x\}
\end{aligned}
$$


Dado $x \in[0,1]$, é importante notar que os dígitos da expansão decimal de $x$ estão relacionados com os intervalos aos quais pertencem os seus iterados da transformação $g$. Por exemplo, se $x=0,3492235 \ldots$, temos

- $x \in[0,3,0,4) \Rightarrow 1^{\circ}$ dígito de sua expansão decimal é 3;

- $g(x) \in[0,4,0,5) \Rightarrow 2^{\circ}$ dígito de sua expansão decimal é 4;

- $g^{2}(x) \in[0,9,1) \Rightarrow 3^{\circ}$ dígito é 9 , e assim sucessivamente.

Figura 1 - Gráfico da transformação $g$.

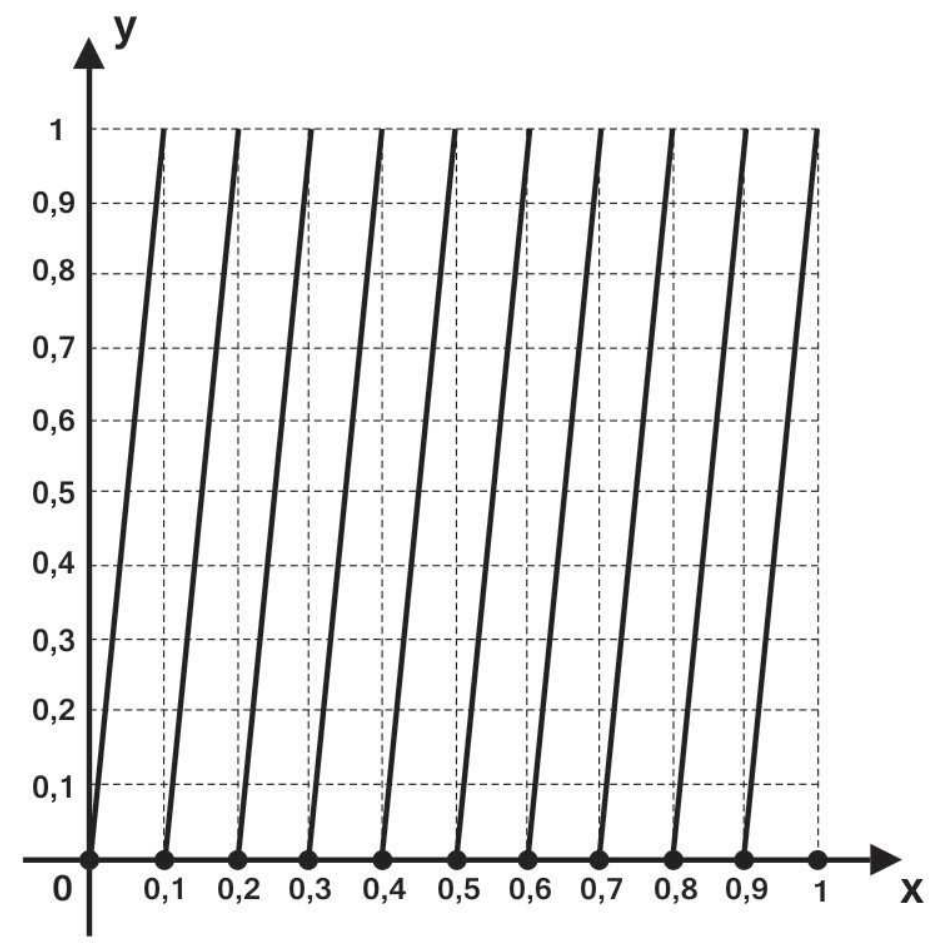

Fonte: Elaboração do autor.

Mais precisamente, temos que 0 -ésimo dígito na expansão decimal do número $x$ é determinado pelo intervalo a que pertence $g^{j-1}(x)$. Em outras palavras, saber se o dígito 7 volta a aparecer na expansão decimal de $x \in[0,7,0,8)$ equivale a saber se existe $j \in \mathbb{N}$ tal que $g^{j}(x) \in[0,7,0,8)$. É claro que estamos usando o dígito 7 apenas para fixar ideias. A mesma argumentação poderia ser usada para quaisquer outros dígitos. 


\section{Estimando Probabilidades via Teoria Ergódica}

O ponto central é que podemos estimar a probabilidade de certos subconjuntos de números reais por meio de ferramentas da Teoria Ergódica. Ao leitor interessado em uma discussão mais aprofundada nessa área, veja Mañé [6], Oliveira e Viana [8], Brown [1], entre outros.

Vamos trabalhar em seguida com algumas definições próprias de Teoria da Medida, como espaço de medida e conjunto mensurável. Resumidamente, um espaço de medida é uma trinca $(M, \beta, \mu)$, em que $M$ é um conjunto, $\beta$ é uma $\sigma$-álgebra e $\mu: \beta \rightarrow \mathbb{R}$ é uma medida. Dizemos também que $B \subset M$ é um conjunto mensurável se $B \in \beta$, ou seja, faz sentido considerar $\mu(B)$. Uma apresentação formal dessas noções pode ser encontrada em Castro Jr. [3] e Isnard [4].

Agora, considere os seguintes conjuntos:

$$
\begin{aligned}
C_{7} & =\{x \in[0,7,0,8) \mid \text { o dígito } 7 \text { aparece novamente na expansão decimal }\} \\
C_{7}^{\infty} & =\{x \in[0,7,0,8) \mid \text { o dígito } 7 \text { aparece infinitas vezes na expansão decimal }\}
\end{aligned}
$$

Nesta seção, vamos mostrar que esses subconjuntos tem medida total de Lebesgue no intervalo considerado. Começamos apresentando a noção de medida invariante:

Definição 2.1. Sejam $(M, \beta, \mu)$ um espaço de medida, e $f: M \rightarrow M$ uma transformação mensurável. Dizemos que a medida $\mu$ é invariante por $f$ se $\mu(E)=\mu\left(f^{-1}(E)\right)$ para todo $E$ mensurável.

Um fato que usaremos a seguir é que a medida de Lebesgue $(\lambda)$ no intervalo $[0,1]$ é invariante pela transformação $g$ (definida na seção anterior). Pelo gráfico da transformação $g$, podemos ver que, se $I \subseteq[0,1]$ é um intervalo, sua pré-imagem é formada por 10 intervalos disjuntos com medida $\frac{1}{10}$ do seu comprimento, e assim $\lambda(I)=\lambda\left(g^{-1}(I)\right)$. Por um argumento típico de Teoria da Medida (veja por exemplo [8]), essa propriedade pode ser estendida para qualquer conjunto mensurável, e assim concluímos que $\lambda$ é invariante por $g$.

Usando este fato, nosso problema de estimar a probabilidade dos conjuntos $C_{7}$ e $C_{7}^{\infty}$ pode ser resolvido por meio de um resultado de Teoria Ergódica, enunciado e demonstrado a seguir:

Teorema 2.2. (Teorema de Recorrência de Poincaré) Sejam $f: M \rightarrow M$ uma transformação mensurável, e $\mu$ uma medida invariante finita. Então para todo $E \subseteq M$ mensurável $(\operatorname{com} \mu(E)>0) \mathrm{e}$ para quase todo $x \in E$ existem infinitos $n \in \mathbb{N}$ tais que $f^{n}(x) \in E$. 
Demonstração. Seja $E \subseteq M$ mensurável. Inicialmente, considere o conjunto dos elementos de $E$ cujos iterados da transformação não retornam a esse conjunto, ou seja:

$$
E_{0}=\left\{x \in E: f^{n}(x) \notin E \forall n \geq 1\right\}
$$

Queremos mostrar que $\mu\left(E_{0}\right)=0$, donde segue que o conjunto dos pontos que retorna a $E$ tem medida total. Defina, para $n \in \mathbb{N}$, o conjunto $E_{n}=f^{-n}\left(E_{0}\right)$. Como $\mu$ é medida invariante, segue por definição que $\mu\left(E_{n}\right)=\mu\left(E_{0}\right)$ para todo $n \in \mathbb{N}$.

Além disso, afirmamos que os conjuntos $E_{n}$ são disjuntos dois a dois. De fato, suponha por absurdo que existe $x \in E_{j} \cap E_{k}$, com $k>j$. Então teríamos $f^{j}(x) \in E_{0}$ e $f^{k}(x) \in E_{0}$. Disso decorre que $f^{k-j}\left(f^{j}(x)\right) \in E_{0}$, o que contradiz a definição de $E_{0}$. Portanto, obtemos uma família $\left\{E_{n}\right\}_{n \in \mathbb{N}}$ de conjuntos disjuntos com a mesma medida. Como $\mu$ é finita por hipótese, a única possibilidade é $\mu\left(E_{n}\right)=\mu\left(E_{0}\right)=0$.

Agora, vamos mostrar que quase todo ponto do conjunto $E$ volta infinitas vezes a esse conjunto. Para tanto, considere: $F=\left\{x \in E: f^{n}(x) \in E\right.$ apenas para uma quantidade finita de n's $\}$. Dado $x \in F$, e tomando o maior dos iterados que retornam ao conjunto $E$, temos que existe $k \in \mathbb{N}$ tal que $f^{k}(x) \in E_{0}$. Dessa forma, $F \subseteq \bigcup_{k=0}^{\infty} f^{-k}\left(E_{0}\right)$. E assim, usando resultados básicos de Teoria da Medida, obtemos:

$$
\mu(F) \leq \mu\left(\bigcup_{k=0}^{\infty} f^{-k}\left(E_{0}\right)\right) \leq \sum_{k=0}^{\infty} \mu\left(f^{-k}\left(E_{0}\right)\right)=\sum_{k=0}^{\infty} \mu\left(E_{0}\right)=0 .
$$

Segundo o Teorema de Recorrência de Poincaré, quase todo ponto de $E$ retorna ao conjunto após um certo número de iterados da transformação $f$ (Figura 2).

Figura 2 - Representação gráfica do Teorema de Recorrência de Poincaré.

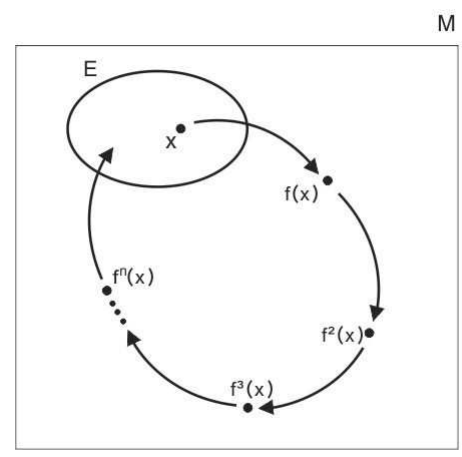

Fonte: Elaboração do autor. 
É interessante constatar que os passos da demonstração acima correspondem aos dois primeiros questionamentos da seção inicial, tomando $E=[0,7,0,8)$. Inicialmente, $\mu\left(E_{0}\right)=0$ nos permite concluir que quase todo elemento desse intervalo vai apresentar novamente o dígito 7 em sua expansão decimal. Em outras palavras, escolhido um elemento de $E$ ao acaso, a probabilidade deste elemento apresentar novamente o dígito 7 em sua expansão decimal é de $100 \%$ (o conjunto $C_{7}$ é de medida total).

Ademais, o fato que $\mu(F)=0$ nos permite ir mais além. Isso significa precisamente que, escolhido um número ao acaso no intervalo $E=[0,7,0,8)$, a chance desse número apresentar o dígito 7 infinitas vezes em sua expansão decimal é de $100 \%$ (o conjunto $C_{7}^{\infty}$, apesar de mais restrito, também é de medida total).

\section{A Frequência de um Dígito na Expansão Decimal}

Além dos conjuntos $C_{7}$ e $C_{7}^{\infty}$, estamos interessados em estimar a medida do conjunto dos números normais. Como mencionamos anteriormente, a propriedade que caracteriza esses números está ligada à sua expansão em casas decimais.

Nesse sentido, um número normal é aquele em que todos os possíveis blocos de $k$ algarismos aparecem com a frequência $\frac{1}{10^{k}}$ em sua expansão decimal, para todo $k \in \mathbb{N}$. Em particular, todos os algarismos de 0 a 9 apresentam a mesma frequência na expansão decimal. Para apresentar a definição com mais rigor, precisamos da noção de frequência de um dígito na expansão decimal, que apresentaremos nesta seção.

Nesse sentido, tome $x \in[0,1]$ e fixe um dígito $i \in\{0,1,2,3,4,5,6,7,8,9\}$. Considere 0 intervalo $I=\left[\frac{i}{10}, \frac{i+1}{10}\right)$. A frequência do dígito $i$ na expansão decimal de $x$ consiste na média de visitas ao conjunto $I$ dos iterados de $g(x)$. Formalmente:

$$
f(i, x)=\lim _{n \rightarrow \infty} \frac{1}{n} \#\left\{j=0, \ldots, n-1: g^{j}(x) \in I\right\} .
$$

Seguem alguns exemplos simples de frequência de um dígito na expansão decimal:

- $f\left(3, \frac{1}{3}\right)=1$, pois $\frac{1}{3}=0,333333 \ldots$, e assim somente o dígito 3 aparece na expansão decimal correspondente;

- $f\left(2, \frac{1}{4}\right)=0$, pois $\frac{1}{4}=0,25$, e como o dígito 2 somente aparece uma vez na expansão decimal, temos $\lim _{n \rightarrow \infty} \frac{1}{n}=0$; 
- $f\left(7, \frac{78}{99}\right)=\frac{1}{2}$, pois $\frac{78}{99}=0,78787878 \ldots$, e assim o dígito 7 aparece metade das vezes na expansão decimal correspondente.

Um problema interessante do ponto de vista matemático trata da existência do limite acima. De fato, é falso afirmar que o limite existe para todo número real no intervalo $[0,1]$. Não é difícil construir um exemplo de um número no qual o limite não está bem definido para algum algarismo. A esse respeito, tome $x \in[0,1]$ tal que:

- Os 10 primeiros algarismos em sua expansão decimal são 7;

- Os $10^{2}$ algarismos seguintes em sua expansão decimal são 0 ;

- Os $10^{3}$ algarismos seguintes em sua expansão decimal são 7;

- Os $10^{4}$ algarismos seguintes em sua expansão decimal são 0 , e assim sucessivamente.

Basicamente, a ideia do padrão de formação de $x$ é escrever os algarismos 0 e 7 em quantidades cada vez maiores para que os termos da sequência:

$$
\left(\frac{1}{n} \#\left\{j=0, \ldots, n-1: g^{j}(x) \in[0,7,0,8)\right\}\right)_{n \in \mathbb{N}}
$$

oscilem de forma significativa, com termos de indíce arbitrariamente grandes abaixo de 0,2 e acima de 0,8 , implicando que o limite correspondente não exista para o algarismo 7 . No entanto, apesar desses contra-exemplos, um resultado de teoria ergódica garante a existência do limite quase sempre (isto é, para um conjunto de medida total). Este resultado, que enunciamos a seguir, é uma versão simplificada do Teorema Ergódico de Birkhoff $^{2}$ para tempos de visita:

Teorema 3.1. (Birkhoff) Seja $f: M \rightarrow M$ uma transformação, e $\mu$ uma medida finita invariante. Seja $E \subseteq M$ um conjunto mensurável. Então, o tempo médio de visita dado por:

$$
\tau(E, x)=\lim _{n \rightarrow \infty} \frac{1}{n} \#\left\{j=0,1, \ldots, n-1: f^{j}(x) \in E\right\}
$$

existe para quase todo $x \in M$.

E, de fato, vimos na Seção 2 que a medida de Lebesgue é invariante pela transformação $g$. Assim, podemos aplicar o teorema para garantir, a menos de um conjunto de medida nula, que a frequência de um dígito na expansão decimal de um número real no intevalo $[0,1]$ está bem definida.

\footnotetext{
${ }^{2}$ Uma discussão sobre esta versão do Teorema pode ser encontrada em Oliveira e Viana [8]. Para mais detalhes sobre o Teorema Ergódico de Birkhoff e sua demonstração, veja Mañé [6] ou Brown [1].
} 
Da mesma forma, podemos considerar blocos de $k$ dígitos, e definir de forma análoga a frequência desses blocos na expansão decimal de um número real. Por exemplo, se estamos interessados no bloco '23', consideramos o intervalo $I=[0,23,0,24)$, e assim sucessivamente. Da mesma forma, esta versão do Teorema de Birkhoff garante a boa definição dessa frequência, a menos de um conjunto de medida nula.

\section{O Subconjunto dos Números Normais}

Com a noção de frequência de um dígito apresentada na seção anterior, podemos definir o conjunto dos números normais de forma precisa. Nesse sentido:

Definição 4.1. Um número $x \in \mathbb{R}$ é dito normal se, dado $k \in \mathbb{N}$, qualquer bloco de $k$ algarismos ocorre com frequência de $\frac{1}{10^{k}}$ em sua expansão decimal.

Uma propriedade inicial é que o conjunto dos números normais é um subconjunto dos irracionais. De fato, sabemos que os números racionais são caracterizados pela periodicidade de sua expansão decimal. Dessa forma, se $x \in \mathbb{Q}$, existe $k \in \mathbb{N}$ e um bloco de $k$ dígitos que não aparece na parte periódica de $x$. Esse bloco possui frequência nula, ou seja, $x$ não é número normal.

Ademais, é fácil verificar que a recíproca não é verdadeira, ou seja, existem números irracionais que não são normais, como todos os números irracionais que não possuem o dígito 3 em sua expansão decimal, por exemplo.

Não é uma tarefa simples exibir exemplos de números normais. Isso porque, para provar formalmente que um determinado número é normal, é preciso verificar a frequência de todos os blocos de $k$ dígitos, com $k \in \mathbb{N}$. Um exemplo é a constante de Champernowne [2], dada por:

$$
\alpha=0,1234567891011121314 \ldots,
$$

que é obtida listando a sequência dos números naturais na expansão decimal. Mais detalhes sobre a construção de outros números normais e demonstrações nesta temática podem ser obtidos em Mengue e Ripoll [7].

A seguir, nosso objetivo é mostrar o conjunto dos números normais continua sendo de medida total (mesmo sendo mais restrito que os irracionais). Em outras palavras, vale o fato surpreendente que, escolhendo ao acaso um número real no intervalo $[0,1]$, a probabilidade deste número ser normal é de $100 \%$. 


\section{Sistemas Ergódicos}

Nesta seção, vamos apresentar alguns resultados de Teoria Ergódica que permitem obter o resultado descrito acima em relação ao conjunto dos números normais. Para tanto, precisamos inicialmente da noção de sistema ergódico. Nesse sentido:

Definição 5.1. Sejam $f: M \rightarrow M$ uma transformação mensurável, e $\mu$ medida de probabilidade invariante por $f$. Dizemos que o sistema $(f, \mu)$ é ergódico se dado $A \subset M$ subconjunto invariante por $f$, temos $\mu(A)=1$ ou $\mu(A)=0$.

Nesse sentido, um exemplo simples de sistema não ergódico é dado por $(I d, \lambda)$, onde $I d$ : $[0,1] \mapsto[0,1]$ é a identidade, e $\lambda$ é a medida de Lebesgue. De fato, todo $E \subseteq[0,1]$ é invariante, pois na transformação $I d$ todo ponto é levado em si mesmo. E, por definição, a existência de conjuntos invariantes não triviais faz com que o sistema não seja ergódico.

Dados uma transformação $f: M \rightarrow M$, um ponto $x \in M$ e um subconjunto $E \subset M$, lembre que $\tau(E, x)$ é o tempo médio de visita ao conjunto $E$ dos iterados de $x$ por $f$. Apresentamos a seguir uma caracterização bastante útil de sistema ergódico em relação aos tempos de visita correspondentes, cuja demonstração pode ser encontrada em Oliveira e Viana [8]:

Proposição 5.2. Sejam $f: M \rightarrow M$ uma transformação mensurável, e $\mu$ medida de probabilidade invariante. Então o sistema $(f, \mu)$ é ergódico se e somente se para todo $E \subseteq M$ mensurável, vale a relação $\tau(E, x)=\mu(E)$ para quase todo $x \in M$.

Finalmente, vamos precisar também da noção de ponto de densidade de um conjunto em Teoria da Medida, que relembramos a seguir:

Definição 5.3. Seja $E \subseteq \mathbb{R}$ mensurável segundo a medida de Lebesgue $\lambda$. Temos que $a \in \mathbb{R}$ é ponto de densidade de $E$ se $\lim _{n \rightarrow \infty} \frac{\lambda\left(E \cap I_{n}\right)}{\lambda\left(I_{n}\right)}=1$, onde $I_{n}$ são intervalos abertos contendo $a$ para todo $n \in \mathbb{N}$, e além disso $\lambda\left(I_{n}\right) \rightarrow 0$ quando $n \rightarrow \infty$.

Vale destacar que a existência desses pontos é garantida pelo resultado que enunciamos a seguir, que é uma consequência do Teorema de Derivação de Lebesgue (mais detalhes sobre o assunto, bem como as demonstrações podem ser encontradas em Castro [3]):

Teorema 5.4. Seja $E \subseteq \mathbb{R}$ mensurável segundo a medida de Lebesgue, $\operatorname{com} \lambda(E)>0$. Então, quase todo ponto de $E$ é ponto de densidade de $E$. 
Usando essas ferramentas, vamos mostrar que o sistema $(g, \lambda)$ é ergódico, onde $g$ é a transformação que definimos na seção inicial, e $\lambda$ é a medida de Lebesgue.

Inicialmente, considere um conjunto invariante $A \subseteq[0,1]$ em relação à transformação $g$, com $\lambda(A)>0$. Vamos mostrar que $\lambda(A)=1$, donde segue que o sistema é ergódico por definição.

Por absurdo, suponha que $\lambda(A)=k<1$. Fixe um ponto $a$ de densidade de $A$, cuja existência é garantida pelo Teorema de Lebesgue. Vamos supor ainda que $a$ não é da forma $\frac{i}{10^{n}}, \operatorname{com} i=$ $0,1,2, \ldots, 10^{n}$ para todo $n \in \mathbb{N}$. Essa hipótese adicional é aceitável, pois $\lambda(A)>0$ e o conjunto dos pontos da forma $\left\{\frac{i}{10^{n}} \mid i=0,1,2, \ldots, 10^{n}\right\}$ é de medida nula, pois é enumerável.

Agora, dado $n \in \mathbb{N}$, defina $I_{n}$ como o intervalo da forma $\left(\frac{i-1}{10^{n}}, \frac{i}{10^{n}}\right)$ que contém o ponto $a$. Pela definição de ponto de densidade, temos:

$$
\lim _{n \rightarrow \infty} \frac{\lambda\left(A \cap I_{n}\right)}{\lambda\left(I_{n}\right)}=1
$$

Assim, pela definição de limite, existe $n$ tal que $\frac{\lambda\left(A \cap I_{n}\right)}{\lambda\left(I_{n}\right)}>k$. Usando que $A$ é invariante em relação a $g$, e que a transformação $g^{n}$ amplia $10^{n}$ vezes um intervalo da forma $\left(\frac{i-1}{10^{n}}, \frac{i}{10^{n}}\right)$, podemos escrever:

$$
k<\frac{\lambda\left(A \cap I_{n}\right)}{\lambda\left(I_{n}\right)}=\frac{\frac{\lambda\left(g^{n}\left(A \cap I_{n}\right)\right)}{10^{n}}}{\frac{1}{10^{n}}}=\lambda\left(g^{n}\left(A \cap I_{n}\right)\right) \leq \lambda\left(g^{n}(A)\right) \leq \lambda(A) .
$$

Da expressão acima, obtemos $\lambda(A)>k$, gerando uma contradição. Portanto, concluímos que $\lambda(A)=1$, e assim o sistema $(g, \lambda)$ é ergódico.

\section{Conclusões e Comentários Finais}

O fato que o sistema $(g, \lambda)$ é ergódico nos permite obter importantes conclusões sobre a frequência dos dígitos na expansão decimal dos números no intervalo $[0,1]$.

Em relação ao dígito 7, a ergodicidade do sistema implica, pela Proposição 5.2, que para quase todo $x \in[0,1]$, temos $\tau(E, x)=\mu(E)=\frac{1}{10}$, em que $E=[0,7,0,8)$. Em outras palavras, quase todos os números reais do intervalo $[0,1]$ apresentam frequência do dígito 7 igual a $\frac{1}{10}$. $\mathrm{E}$ obviamente, o mesmo vale para todos os outros dígitos. 
Analogamente, tomando um bloco de $k$ dígitos, segue diretamente da Proposição 5.2 que quase todo número real no intervalo $[0,1]$ apresenta, em sua expansão decimal, frequência desse bloco igual a $\frac{1}{10^{k}}$.

Ora, fixando o dígito (ou bloco de $k$ dígitos), o conjunto dos pontos em que frequência não é a desejada tem medida nula. Usando o resultado simples de Teoria da Medida segundo o qual uma união enumerável de conjuntos de medida nula tem medida nula, obtemos diretamente que o conjunto dos números que não são normais tem medida nula em relação à medida de Lebesgue.

E assim, finalmente respondemos nosso último questionamento inicial, mostrando via Teoria Ergódica o fato que o conjunto dos números normais é de medida total. Em outras palavras, é surpreendente que, apesar da dificuldade de exibir um número normal, se escolhermos ao acaso um número do intervalo $[0,1]$, quase certamente este número será normal.

\section{Referências}

[1] BROWN, J. Ergodic Theory and Topological Dynamics. New York: Academic Press, 1976.

[2] CHAMPERNOWNE, D. The construction of decimals normal in the scale of ten. Journal of the London Mathematical Society. v. 8, p. 254-260, 1933.

[3] CASTRO Jr., A. Curso de Teoria da Medida. Rio de Janeiro: Instituto Nacional de Matemática Pura e Aplicada, 2008.

[4] ISNARD, C. Introdução à Medida e Integração. Rio de Janeiro: Instituto Nacional de Matemática Pura e Aplicada, 2009.

[5] LIMA, E. Curso de Análise. v. 1. Rio de Janeiro: Instituto Nacional de Matemática Pura e Aplicada, 2012.

[6] MAÑÉ, R. Introdução à Teoria Ergódica. Rio de Janeiro: Instituto Nacional de Matemática Pura e Aplicada, 1983.

[7] MENGUE, J.; RYPOLL, C. Introdução aos números normais. COLÓQUIO DE MATEMÁTICA DA REGIÃO SUL, 2., 2012, Londrina. Anais... Sociedade Brasileira de Matemática, 2012.

[8] OLIVEIRA, K.; VIANA, M. Fundamentos da Teoria Ergódica. Rio de Janeiro: Sociedade Brasileira de Matemática, 2014.

[9] TAYLOR, M. Measure Theory and Integration. Graduate Studies in Mathematics. v. 76. American Mathematical Society, 2000. 